\title{
Análisis de instrumentos para el diagnóstico de la competencia digital
}

\section{Analysis of instruments for the diagnosis of digital competence}

\author{
César González-Rodríguez \\ Universitat de les Illes Balears \\ cesargr@educastur.org \\ Santos Urbina-Ramírez \\ Universitat de les Illes Balears \\ santos.urbina@uib.es
}

Recibido: $21 / 01 / 2020$

Aceptado: $21 / 05 / 2020$

Publicado: 1/12/2020

\section{RESUMEN}

La importancia que han cobrado las Tecnologías de la Información y Comunicación en la sociedad durante los últimos años ha provocado que la competencia digital sea considerada como clave en el diseño de las políticas educativas y, en consecuencia, que desde diversos ámbitos se hayan desarrollado múltiples instrumentos destinados a la evaluación de las habilidades y destrezas digitales de docentes, discentes y población en general. Es por ello que se ha considerado pertinente analizar diversos tipos de herramientas usadas en la última década en España para el diagnóstico de la competencia digital del alumnado de distintas etapas educativas prestando atención, entre otros aspectos, a los ítems utilizados, la estructura de las herramientas o la metodología empleada. Este trabajo profundiza en el análisis de una serie de investigaciones que, pese a compartir, en muchos casos, aspectos metodológicos, difieren en su visión y concepción de la competencia digital, algo que dificulta el establecimiento de pautas comunes de evaluación, ya que resulta complicado acordar cómo medir una variable cuando la definición de la misma se presta a múltiples interpretaciones. Precisamente la definición de un marco común de referencia en el ámbito educativo que sirva para abordar la evaluación de las habilidades digitales es uno de los retos de investigadores e instituciones, si bien no se trata de una tarea sencilla cuando las tecnologías digitales se caracterizan por los continuos y vertiginosos cambios.

\section{PALABRAS CLAVE}

Competencia digital; evaluación; instrumento de medida; tecnologías de la información y comunicación.

\section{ABSTRACT}

The impact of Information and Communication Technologies on society in recent years has caused digital competence to be considered the key to designing educational policies and, consequently, the development of numerous instruments for the evaluation of the digital skills and abilities of teachers, students and the population, in general, in several fields. Therefore, it has been considered relevant to analyze various types of tools used in the past decade in Spain for the diagnosis of the digital competence of students from different educational stages, paying attention, among others, to the items used, the structure of the tools or the methodology. This work goes in depth in the analysis of a series investigations that, despite sharing some methodological aspects in many ways, differ in their vision and conception of the digital competence. This makes the establishment of some common evaluation guidelines more difficult, since it is complicated to agree on how to measure a variable when its own definition could be interpreted in several ways. The definition of a common frame of reference in the educational field that serves to address the evaluation of 
digital skills is precisely one of the challenges of researchers and institutions. However, it is not an easy task when digital technologies are characterized by continuous and vertiginous changes.

\section{KEYWORDS}

Digital competence; evaluation; measuring instrument; information and communication technologies.

\section{CITA RECOMENDADA}

González-Rodríguez, C. \& Urbina-Ramírez, S. (2020). Análisis de instrumentos para el diagnósticos de la competencia digital. Revista Interuniversitaria de Investigación en Tecnología Educativa, 9, 1-12. http://dx.doi.org/10.6018/riite.411101

\section{Principales aportaciones del artículo y futuras líneas de investigación:}

- Analizar comparativamente diversos instrumentos de medida de la competencia digital utilizados en España en los últimos diez años, así como la evolución de los mismos a lo largo de este tiempo.

- Comprobar la validez de los instrumentos más relevantes analizados en contextos educativos no formales

- Diseñar y validar un instrumento de medida de carácter más práctico para acompañar y/o contrastar los cuestionarios de autoevaluación.

\section{INTRODUCCIÓN}

El desarrollo de las Tecnologías de la Información y Comunicación (TIC) ha provocado grandes cambios que afectan a la configuración socio-económica a escala global y en los que se enmarca la denominada tercera revolución industrial. Sin apenas tiempo para digerir las importantes transformaciones de las últimas décadas, Galindo, Ruiz y Ruiz (2017) auguran que elementos como el Internet de las cosas, la automatización, la robotización o la inteligencia artificial abren la puerta a una cuarta revolución industrial caracterizada por cambios aún más vertiginosos.

Resulta lógico, por tanto, que la preocupación por el nivel de competencia digital de la ciudadanía se haya incrementado notablemente, algo que se evidencia en la proliferación de literatura científica en los últimos años o en el desarrollo de estrategias e iniciativas institucionales encaminadas a la adquisición de habilidades en el uso de las TIC (Prendes et al., 2010). Buena parte de estas acciones se han vinculado al ámbito educativo y, de hecho, la Comisión Europea (2006), en su Recomendación sobre las competencias clave para el aprendizaje permanente (2006/962/CE), consideran la competencia digital como una de las ocho competencias clave e inciden en la importancia del dominio de las denominadas tecnologías de la sociedad de la información a la hora de afrontar "situaciones cotidianas de la vida privada, social y profesional" (p. 16).

Además del cambio motivado por la adopción de un nuevo modelo educativo en la Unión Europea, basado en las competencias y culminado con el desarrollo del Espacio Europeo de Educación Superior (EEES), otras instituciones internacionales como la UNESCO y la OCDE han pilotado proyectos encaminados al desarrollo de nuevos procesos de enseñanza-aprendizaje que están relacionados con el concepto de competencia y que tienen en cuenta las nuevas posibilidades ofrecidas por las TIC (Larraz et al., 2013).

Esta novedosa visión de las políticas educativas ha tenido su impacto en España, siendo la experiencia más significativa el programa Escuela 2.0, puesto en marcha en 2010 por parte del Ministerio de Educación, Cultura y Deporte para la inclusión de las tecnologías digitales en el aula (González et al., 2012). La iniciativa abordaba la formación del profesorado, la creación de 
contenidos digitales y el uso responsable de las herramientas tecnológicas mediante el uso de portátiles y miniordenadores en las clases de Educación Primaria.

No es de extrañar que el protagonismo adquirido por las TIC haya provocado un interés creciente por la evaluación de las habilidades digitales, si bien, antes de profundizar en el análisis de los instrumentos diagnósticos, resulta conveniente centrarse en el objeto de medida. Así pues, ¿qué se entiende por competencia digital? ¿Existe unanimidad a la hora de definir este concepto?

\subsection{Concepto de competencia digital}

El concepto de competencia digital ofrece múltiples matices y resulta complicado encontrar un marco de referencia común que sirva de punto de partida para el diseño de políticas, estrategias y acciones, así como para su posterior evaluación. De Pablos et al. (2016) inciden en esta dificultad y citan a Van Deursen y Van Dijk (2009), cuyos trabajos sobre el marco conceptual de la competencia digital concluyen que buena parte de las definiciones son superficiales, pues se limitan a considerar habilidades vinculadas al uso de ordenadores e Internet. En el contexto descrito, la ya citada Recomendación 2006/962/CE de la Comisión Europea (2006) ofrece una visión más amplia con la siguiente definición:

La competencia digital entraña el uso seguro y crítico de las tecnologías de la sociedad de la información (TSI) para el trabajo, el ocio y la comunicación. Se sustenta en las competencias básicas en materia de TIC: el uso de ordenadores para obtener, evaluar, almacenar, producir, presentar e intercambiar información, y comunicarse y participar en redes de colaboración a través de Internet. (p. 15)

Igualmente la Internacional Society for Technology in Education (ISTE) se refiere en 2007 a "lo que los estudiantes deberían saber y ser capaces de hacer para aprender efectivamente y vivir productivamente en un mundo cada vez más digital" y fija estándares en los siguientes apartados: creatividad e innovación; comunicación y colaboración; investigación y manejo de información; pensamiento crítico, solución de problemas y toma de decisiones; ciudadanía digital; funcionamiento y conceptos de las TIC. En una línea similar, Ala-Mutka (2011) señala que la competencia digital debe considerarse como un concepto más amplio que la mera capacidad de utilizar herramientas y medios digitales, incidiendo además en la importancia de las "actitudes interculturales, críticas, creativas, responsables y autónomas". (p. 53)

El Joint Research Centre (JRC), organismo de investigación de la Unión Europea, da continuidad al trabajo de Ala-Mutka y desarrolla el Marco de Competencia Digital, conocido por el acrónimo de DigComp. Su primera versión se publica en 2013 y es actualizada en 2016 en la forma DigComp 2.0 con la presentación de 21 competencias, que posteriormente son agrupadas en ocho niveles con ejemplos de uso en DigComp 2.1 (Carretero et al, 2017). La intención inicial de esta iniciativa es la de mejorar la competencia digital de la población a través del desarrollo de políticas específicas y estableciendo un marco de referencia común a nivel europeo (Vuorikari et al., 2016).

\subsection{Evaluación de la competencia digital}

Las estrategias diseñadas por las instituciones para el desarrollo de habilidades digitales precisan de instrumentos que permitan evaluar el nivel de competencia digital, no sólo para constatar la adquisición de los correspondientes conocimientos y capacidades en cada etapa educativa, sino también para diseñar procesos formativos adecuados para dicha tarea (Gisbert y Esteve, 2011; González et al., 2012). Pech y Prieto (2016) destacan alguna de las primeras propuestas dirigidas a la evaluación de la competencia digital como el sistema de certificaciones C2i y C2i2e, existente en Francia desde 2000 para verificar las competencias en informática e Internet, la certificación MITIC del cantón suizo de Ginebra o diversas iniciativas implementadas en Estados Unidos como son los estándares ILSHE, los estándares NETS de la ISTE y el Test de Beile de conocimientos de Informática para la Educación (BTILE).

Si los instrumentos desarrollados en los últimos años se caracterizan por su diversidad y variedad de enfoques, los estándares definidos a través de DigComp pueden contribuir a unificar criterios de evaluación y, de hecho, se tiene en cuenta en alguno de los trabajos de diagnóstico 
más recientes efectuados en España (Colás et al. 2017; González et al., 2018; Pérez, 2015). En cualquier caso, resulta pertinente el análisis de instrumentos de medida de la competencia digital utilizados en los últimos años para evaluar las habilidades y destrezas de alumnado de diversas etapas educativas.

\section{PROCEDIMIENTO}

El trabajo presentado tiene como objetivo localizar y analizar diferentes herramientas de corte cuantitativo para el diagnóstico de la competencia digital de estudiantes. Para ello, se realiza una revisión documental atendiendo a criterios de impacto en plataformas como Dialnet, Google Scholar, Scopus y Web of Science siguiendo las recomendaciones de González y Román (2016) para la búsqueda bibliográfica. La misma se vertebra sobre la palabra clave "competencia digital", y su traducción al inglés "digital competence", en combinación con operadores booleanos AND para añadir "evaluación", "análisis" o "diagnóstico". También se utiliza el operador NOT para excluir "docente", puesto que predominan los trabajos que abordan la competencia digital docente sobre los relativos a alumnado.

La búsqueda se limita a instrumentos diseñados en España en el marco temporal de la última década. Para el cribado, se valoran los siguientes aspectos: marco de referencia teórico, claridad en los objetivos planteados, utilización de procedimientos de validación, dimensión de los estudios asociados, impacto de las publicaciones difundidas, accesibilidad a los datos y consistencia de los mismos. Igualmente se tiene en cuenta la diversidad de enfoques investigadores y la variedad de contextos educativos existentes para limitar el riesgo de sesgo en un estudio de estas características.

Tras cruzar los resultados obtenidos en los buscadores señalados, el primer cribado da como resultado el acceso a 39 investigaciones distintas, de las que inicialmente se descartan 8 por no referirse al contexto español y otras 3 por tratarse de estudios cualitativos. A su vez, se prescinde de otros 5 trabajos por tratarse de estudios sectoriales dirigidos a mayores o mujeres. Para la selección final, las principales causas de exclusión son: utilización de muestras pequeñas, falta de validación de los cuestionarios, dificultad de acceso a los ítems y/o cuestionario, enfoque muy orientado hacia futuras prácticas profesionales y falta de coherencia en los datos presentados.

\section{RESULTADOS}

En base a los criterios definidos, se seleccionan 11 instrumentos de evaluación, de los que se obtiene una serie de datos que son procesados mediante la correspondiente reducción, disposición y agrupamiento (Rodríguez et al., 2005). Esta labor facilita la presentación de cada uno de ellos en los siguientes apartados, así como la confección de la Tabla 1 con los elementos más significativos

\subsection{Inventario de Competencias TIC para estudios de Grado (INCOTIC-Grado)}

Se desarrolla con el objetivo de planificar la docencia en materia de TIC en función del nivel de competencia digital de estudiantes que acceden a la Universitat Rovira i Virgili (González et al., 2010). Parte del trabajo realizado en la misma universidad dentro del proyecto de innovación CREDEFIS (Centro de Recursos Virtual para la Docencia de calidad en Educación Física), cuyo cuestionario fue adaptado posteriormente para el diseño de la herramienta INFERTIC-alumnos dirigido a estudiantes del último curso de Enfermería de la URV (Gisbert et al., 2011).

El cuestionario, además de los datos identificativos iniciales, incluye apartados relativos a la disponibilidad de recursos, uso real, formación, valoración y actitudes hacia las TIC. Alguno de los estudios realizados con este instrumento resalta el alto nivel de competencia digital, especialmente a la hora de navegar por Internet o de usar herramientas ofimáticas, si bien se considera necesario reforzar la capacidad de análisis crítico en la búsqueda de información o la utilización de programas más específicos del ámbito educativo (Espuny et al, 2010; Larraz, 2013). 
Se ha de destacar que este instrumento es uno de los primeros intentos realizados en España para la medida del nivel de competencia digital y se convierte en un elemento de referencia en este ámbito tras la puesta en marcha del EEES. No obstante, en 2016 se diseña el cuestionario INCOTIC 2.0 como versión actualizada que se ajusta a la definición de competencia digital dada por Larraz (2013) y que además introduce mejoras técnicas para resolver alguna de las deficiencias detectadas tras un lustro de uso (González et al., 2018).

\subsection{Inventario de Competencias TIC para Educación Secundaria Obligatoria (INCOTIC-ESO)}

El interés por conocer el nivel de competencia digital del alumnado que accede a la Educación Secundaria Obligatoria (ESO) motiva la adaptación de INCOTIC a dicha etapa. Además de buscar una introducción ordenada de las TIC en los contenidos curriculares, se pretende recoger evidencias del grado de adquisición de las habilidades digitales durante la ESO en un contexto marcado por la puesta en marcha del programa Escuela 2.0 (González et al., 2012). Se incluyen apartados sobre recursos digitales, grado de uso y conocimiento, cultura y respeto en el uso de información digital, acceso eficiente a la información y comunicación de la misma.

Este cuestionario es utilizado por González (2012) y Fernández (2018) para evaluar respectivamente el impacto de experiencias como eduCAT 1x1 (Cataluña) y proyecto 1:1 (Región de Murcia), caracterizadas por la introducción de un ordenador por estudiante en las aulas de la ESO. En ambos casos, se señala que el impacto de las iniciativas contribuye positivamente al desarrollo de la competencia digital del alumnado.

\subsection{Cuestionario de evaluación de competencias digitales}

Tomando como referencia el estudio de Competencias básicas en el uso de las TIC (COMPETIC), coordinado por el Consejo Superior de Evaluación del Sistema Educativo de la Generalitat de Catalunya, desde la Universitat de Lleida, Carrera et al. (2011) diseñan un instrumento para la evaluación de las habilidades digitales de jóvenes en situación de riesgo desde la hipótesis de que la baja competencia digital puede ser un elemento que contribuya a la exclusión social.

La herramienta utilizada es un extenso cuestionario que se estructura en seis apartados: datos personales, información general, utilización de dispositivos digitales, utilización de aplicaciones multiplataforma, información y comunicación en red y actitudes ante las TIC. La investigación más significativa realizada con este instrumento es dirigida por Vaquero (2013), quien concluye que los jóvenes en situación de riesgo tienen una autopercepción más baja de su competencia digital.

\subsection{Competencias básicas digitales de 2.0 de los estudiantes universitarios (COBADI)}

En el marco de un proyecto participado por investigadores de la Universidad de Alicante, la Universidad de Valencia y la Universidad Pablo Olavide de Sevilla, se diseña un cuestionario de autoevaluación que pretende aprovechar y optimizar los recursos digitales en el aula partiendo del conocimiento del nivel de competencia digital del alumnado (Roig et al., 2012).

Sigue un enfoque similar al INCOTIC con cuestiones relativas al uso general de Internet y de las TIC para la comunicación, colaboración, búsqueda y manejo de información, además de incluir apartados específicos sobre el papel que juegan las TIC en la universidad. Este instrumento se utiliza en 2012 para la realización de un estudio con alumnado de la Universidad de Alicante y se aprecia un buen nivel de competencia en la navegación por Internet y el trabajo con documentos en la red, aunque se encuentran carencias en la utilización de mapas conceptuales para la organización de la información y en el uso de herramientas de software social. 


\subsection{Cuestionario sobre actitud, conocimiento y uso de TIC (ACUTIC)}

El cambio de modelo educativo impuesto por la creación del EEES impulsa el diseño de este cuestionario en 2012 con el objetivo de instrumentos válidos y fiables para la evaluación de la competencia digital (Mirete et al., 2015). Este instrumento desarrollado en la Universidad de Murcia se divide en tres apartados relativos a actitud, conocimiento y uso de las TIC y es sometido a un proceso de contraste de fiabilidad que se realiza con una muestra de 1906 estudiantes durante el curso 2012/2013.

\subsection{Cuestionario de Autopercepción de la Competencia Digital Docente (CACDD)}

Si el objeto de los anteriores cuestionarios era el diagnóstico del nivel de competencia digital, de cara a mejorar la planificación educativa en lo referente a la adquisición de destrezas y habilidades en materia de TIC, este instrumento se diseña desde la perspectiva de que los estudiantes serán futuros profesionales y precisarán un nivel de competencia digital adecuado para el desempeño de su actividad profesional en un entorno altamente digitalizado (Gisbert y Esteve, 2011). Se utiliza como referencia la rúbrica ISTE para profesorado y se incluyen apartados sobre aprendizaje y creatividad del alumnado, experiencias de aprendizaje y evaluación, trabajo y aprendizaje en la era digital, ciudadanía digital y responsabilidad y crecimiento profesional y liderazgo.

Esteve (2015) utiliza este instrumento en una investigación en 2014 con estudiantes de la Facultad de Ciencias de la Educación y Psicología de la Universitat Rovira y Virgili para diagnosticar la competencia digital docente de futuros profesionales. La valoración que el alumnado hace de su propia competencia docente es buena, especialmente en materia de habilidades digitales básicas, si bien los resultados son más modestos en los apartados correspondientes a competencias didácticas con TIC y aquellos que tienen que ver con estrategias para el crecimiento profesional.

\subsection{ETeach3D}

Como ya se ha comentado, el cuestionario de autoevaluación es el tipo de instrumento más utilizado para el diagnóstico de la competencia digital. Sin embargo, a nivel internacional, tal y como destacan Esteve y Gisbert (2013), están desarrollados estándares de competencia como el Instant Digital Competence Assessment (iDCA), el International Computer Driving License (ICDL), el iSkills Assessment o el Programa para la Evaluación Internacional de Estudiantes (PISA), en su apartado dedicado a las TIC, que abordan la evaluación con pruebas de aptitud e incluso con la ejecución de actividades.

Esteve (2015) explora las posibilidades de distintas herramientas prácticas para la evaluación de las habilidades relacionadas con las TIC y apuesta por un entorno virtual 3D para conseguir abordar todas las dimensiones de la competencia digital. Su novedosa propuesta se sustancia en un instrumento dirigido a estudiantes del Grado de Educación Primaria y de Pedagogía en el que se recrean situaciones que se dan en un aula y cuya evaluación se realiza mediante la verificación de 40 indicadores, partiendo de los estándares ISTE utilizados para el diseño de CACDD. En una misma muestra, el uso del ETeach3D y del CACDD permite concluir que el nivel de competencia digital que se desprende del entorno virtual es menor el que se deriva a la autopercepción del alumnado.

\subsection{Cuestionario a futuros docentes de la Universidad de Castilla La Mancha}

Este instrumento se centra en el diagnóstico de la competencia digital de alumnado que está finalizando sus estudios universitarios y, por tanto, debe acreditar su capacitación digital para su futuro desarrollo profesional. Para su diseño se realiza una selección de preguntas incluidas en cuestionarios utilizados con anterioridad en trabajos de investigación que tenían por objeto el estudio de la integración de las TIC en instituciones universitarias, además de incorporar nuevas cuestiones vinculadas a las variables que se desean medir (Cózar y Roblizo, 2014; Roblizo y Cózar, 2015).

Esta herramienta diagnóstica incluye ítems relacionados con la creación y edición de documentos y objetos multimedia, la búsqueda y tratamiento de la información, la comunicación 
y colaboración, el uso de las TIC en el ámbito educativo y las tecnologías emergentes. En 2014 se utiliza en el marco de una investigación con alumnado de último curso de Grado en Maestro de Educación Infantil en la Universidad de Castilla La Mancha y que toma una muestra de 224 estudiantes que destacan en la dimensión comunicativa, así como en el tratamiento y difusión de información, mientras que en el uso de recursos digitales educativos obtienen peores resultados.

\subsection{Autoevaluación de la competencia digital alumnado no universitario}

La mayor parte de las herramientas recopiladas se dirigen hacia alumnado universitario y, por ello, se ha de destacar la propuesta de Colás et al. (2017), quienes se hacen eco de las recomendaciones de organismos internacionales sobre la conveniencia de evaluar la competencia digital del alumnado de Primaria y Secundaria y de incidir en el desarrollo de iniciativas que impulsan la incorporación de las TIC en dichas etapas educativas.

El cuestionario de autoevaluación parte del modelo teórico desarrollado por Ala-Mutka (2011) en el marco del programa DigComp e incluye aspectos relativos al aprendizaje, al uso de herramientas de búsqueda y comunicación o a elementos de seguridad. En coordinación con el Centro de Profesores de Sevilla, este instrumento sirve para la realización de una investigación en centros educativos de primaria y secundaria con trayectoria en la utilización de las TIC en las aulas. Cabe reseñar que se observa diferencias significativas en el nivel de competencia digital en función de la disponibilidad de Internet en el hogar, la utilización de dispositivos portátiles en su centro educativo y la edad de inicio en el uso de ordenador.

\subsection{Cuestionario para la evaluación de la competencia digital en Castilla y León}

Ante la escasez de estudios diagnósticos de la competencia en Educación Primaria, este instrumento pretende conocer el grado de alfabetización digital de una generación que ha nacido en un entorno altamente digitalizado. Pérez (2015) toma como referencia el marco DigComp e incluye ítems relativos a información, comunicación, creación de contenidos, seguridad y resolución de problemas, a los que añade cuestiones relativas al uso de las TIC y su integración en las actividades cotidianas.

El instrumento es utilizado para realizar una investigación entre alumnado de primaria de Castilla y León, sobre una muestra de 674 estudiantes, y paralelamente se evalúa la competencia digital docente del profesorado. Los resultados indican que, pese a tratarse de nativos digitales, el alumnado demuestra una competencia baja y que, por tanto, se ha incidir en los contenidos curriculares relacionados con las TIC.

\subsection{Ikanos Test}

Su diseño es impulsado por el Departamento de Desarrollo Económico e Infraestructuras del Gobierno Vasco y se propone como instrumento de medida de la competencia digital en el marco de un proyecto que pretende aprovechar la potencialidad de las TIC para contribuir al desarrollo de la sociedad. De esta forma, cualquier ciudadano puede acceder al mismo y conocer su perfil digital a partir de un informe personalizado que se obtiene automáticamente tras cumplimentar el test. Para su desarrollo, se toma como referencia la versión 2.1 del marco DigComp (Carretero et al., 2017).

La consistencia de este cuestionario hace que González et al. (2018) lo utilicen para la medida de la competencia digital de alumnado del Grado en Pedagogía de la Universidad de Murcia que cursa la materia "TIC para la formación". Tras utilizar el test al comienzo y final de la asignatura, se aprecian diferencias significativas en todos los apartados, lo que indica que la inclusión de asignaturas específicas sobre las TIC puede tener efectos positivos en la competencia digital del alumnado.

En la siguiente tabla, se realiza un estudio comparativo con los elementos más característicos de las herramientas descritas anteriormente. 
Tabla 1. Principales características de los instrumentos seleccionadas

\begin{tabular}{|c|c|c|c|c|c|c|}
\hline Instrumento & Institución & $\begin{array}{c}\text { Nivel } \\
\text { educativo }\end{array}$ & Tipo & Preguntas & Validación & Soporte \\
\hline $\begin{array}{l}\text { INCOTIC } \\
\text { Grado }\end{array}$ & $\begin{array}{c}\text { Universitat } \\
\text { Rovira i } \\
\text { Virgili } \\
(2009)\end{array}$ & Universidad & $\begin{array}{c}\text { Cuestionario } \\
\text { auto } \\
\text { evaluación } \\
\text { (78 ítems) }\end{array}$ & $\begin{array}{c}\text { Escala } \\
\text { Likert } \\
(1-3 \text { y } 1-5)\end{array}$ & $\begin{array}{l}\text { Muestro } \\
\text { inicial, } \\
\text { panel de } \\
\text { expertos y } \\
\text { validación } \\
\text { final }\end{array}$ & Digital \\
\hline INCOTIC ESO & $\begin{array}{l}\text { Universitat } \\
\text { Rovira i } \\
\text { Virgili } \\
(2010)\end{array}$ & $\begin{array}{l}\text { Educación } \\
\text { Secundaria }\end{array}$ & $\begin{array}{c}\text { Cuestionario } \\
\text { auto } \\
\text { evaluación } \\
\text { (63 ítems) }\end{array}$ & $\begin{array}{c}\text { Escala } \\
\text { Likert } \\
(1-4 \text { y } 1-5)\end{array}$ & $\begin{array}{l}\text { Muestro } \\
\text { inicial, } \\
\text { panel de } \\
\text { expertos y } \\
\text { validación } \\
\text { final }\end{array}$ & Digital \\
\hline $\begin{array}{c}\text { Cuestionario } \\
\text { de evaluación } \\
\text { de } \\
\text { competencias } \\
\text { digitales }\end{array}$ & $\begin{array}{l}\text { Universitat } \\
\text { de Lleida } \\
\text { (2012) }\end{array}$ & $\begin{array}{l}\text { Jóvenes en } \\
\text { riesgo de } \\
\text { exclusión } \\
\text { social (12- } \\
18 \text { años) }\end{array}$ & $\begin{array}{c}\text { Cuestionario } \\
\text { auto } \\
\text { evaluación } \\
\text { (205 ítems) }\end{array}$ & $\begin{array}{c}\text { Escala } \\
\text { Likert (1-5) }\end{array}$ & $\begin{array}{l}\text { Panel de } \\
\text { expertos }\end{array}$ & Papel \\
\hline COBADI & $\begin{array}{c}\text { Universidad } \\
\text { de Alicante, } \\
\text { Universidad } \\
\text { de Valencia } \\
\text { y } \\
\text { Universidad } \\
\text { Pablo } \\
\text { Olavide } \\
\text { (2012) }\end{array}$ & Universidad & $\begin{array}{c}\text { Cuestionario } \\
\text { auto } \\
\text { evaluación } \\
\text { (31 ítems) }\end{array}$ & $\begin{array}{c}\text { Escala } \\
\text { Likert (1-4) }\end{array}$ & $\begin{array}{l}\text { Muestreo } \\
\text { inicial y } \\
\text { panel de } \\
\text { expertos }\end{array}$ & Digital \\
\hline ACUTIC & $\begin{array}{l}\text { Universidad } \\
\text { de Murcia } \\
\text { (2012) }\end{array}$ & Universidad & $\begin{array}{c}\text { Cuestionario } \\
\text { auto } \\
\text { evaluación } \\
\text { (31 ítems) }\end{array}$ & $\begin{array}{c}\text { Escala } \\
\text { Likert } \\
(1-5)\end{array}$ & $\begin{array}{c}\text { Grupo de } \\
\text { discusión y } \\
\text { panel de } \\
\text { expertos }\end{array}$ & Papel \\
\hline CACDD & $\begin{array}{c}\text { Universitat } \\
\text { Rovira i } \\
\text { Virgili } \\
(2014) \\
\end{array}$ & Universidad & $\begin{array}{c}\text { Cuestionario } \\
\text { auto } \\
\text { evaluación } \\
\text { (31 ítems) }\end{array}$ & $\begin{array}{c}\text { Escala } \\
\text { Likert (1-8) }\end{array}$ & $\begin{array}{l}\text { Panel de } \\
\text { expertos }\end{array}$ & Papel \\
\hline ETeach3D & $\begin{array}{l}\text { Universitat } \\
\text { Rovira i } \\
\text { Virgili } \\
\text { (2014) }\end{array}$ & Universidad & $\begin{array}{c}\text { Entorno 3D } \\
\text { de } \\
\text { simulación } \\
\text { de tareas }\end{array}$ & $\begin{array}{c}\text { Checklist } \\
\text { con } 40 \\
\text { apartados }\end{array}$ & $\begin{array}{l}\text { Grupo de } \\
\text { estudiantes } \\
\text { y opinión } \\
\text { de } \\
\text { expertos }\end{array}$ & Digital \\
\hline $\begin{array}{c}\text { Cuestionario } \\
\text { a futuros } \\
\text { docentes de } \\
\text { la UCLM }\end{array}$ & $\begin{array}{c}\text { Universidad } \\
\text { Castilla La } \\
\text { Mancha } \\
(2014)\end{array}$ & Universidad & $\begin{array}{c}\text { Cuestionario } \\
\text { auto } \\
\text { evaluación } \\
\text { (63 ítems) }\end{array}$ & $\begin{array}{c}\text { Escala } \\
\text { Likert }(1-5)\end{array}$ & $\begin{array}{l}\text { Panel de } \\
\text { expertos }\end{array}$ & $\begin{array}{c}\text { No se } \\
\text { especifica }\end{array}$ \\
\hline $\begin{array}{c}\text { Auto } \\
\text { evaluación de } \\
\text { competencia } \\
\text { digital en } \\
\text { alumnado no } \\
\text { universitario } \\
\end{array}$ & $\begin{array}{c}\text { Universidad } \\
\text { de Sevilla } \\
(2014)\end{array}$ & $\begin{array}{l}\text { Educación } \\
\text { primaria y } \\
\text { secundaria }\end{array}$ & $\begin{array}{c}\text { Cuestionario } \\
\text { auto } \\
\text { evaluación } \\
\text { (29 ítems) }\end{array}$ & $\begin{array}{c}\text { Escala } \\
\text { Likert (1-5) }\end{array}$ & $\begin{array}{c}\text { No se } \\
\text { especifica }\end{array}$ & Papel \\
\hline $\begin{array}{c}\text { Cuestionario } \\
\text { competencia } \\
\text { digital en } \\
\text { Castilla y } \\
\text { León }\end{array}$ & $\begin{array}{c}\text { Universidad } \\
\text { de } \\
\text { Salamanca } \\
(2014)\end{array}$ & $\begin{array}{l}\text { Educación } \\
\text { primaria }\end{array}$ & $\begin{array}{c}\text { Cuestionario } \\
\text { auto } \\
\text { evaluación } \\
\text { (44 ítems) }\end{array}$ & $\begin{array}{c}\text { Escala } \\
\text { Likert (1-5) }\end{array}$ & $\begin{array}{c}\text { Aplicación } \\
\text { piloto con } \\
15 \\
\text { alumnos/as }\end{array}$ & Papel \\
\hline IkanosTest & $\begin{array}{c}\text { Gobierno } \\
\text { Vasco } \\
(2017)\end{array}$ & $\begin{array}{l}\text { Población } \\
\text { en general }\end{array}$ & $\begin{array}{c}\text { Cuestionario } \\
\text { auto } \\
\text { evaluación } \\
\text { (32 ítems) }\end{array}$ & $\begin{array}{l}\text { Escala } \\
\text { Likert } \\
(1-10)\end{array}$ & $\begin{array}{l}\text { No se } \\
\text { especifica }\end{array}$ & Digital \\
\hline
\end{tabular}




\section{CONCLUSIONES}

Es innegable que las TIC han entrado de lleno en la vida cotidiana de las personas y, en este contexto, las instituciones educativas están obligadas a diseñar procesos de enseñanzaaprendizaje que desarrollen las habilidades digitales del alumnado. Precisamente del ámbito educativo, y concretamente del universitario, proceden la mayor parte de las herramientas aquí presentadas, siendo las universidades las principales impulsoras de iniciativas dirigidas al diagnóstico de la competencia digital en nuestro país, siguiendo la línea marcada por organismos internacionales y administraciones públicas. De esta forma, comprueban que sus estudiantes adquieren el nivel adecuado de esta competencia y a la vez obtienen datos de utilidad para ajustar sus planes educativos en materia digital.

Sin embargo, tal y como se ha apuntado, la evaluación de la competencia digital es una tarea compleja debido a la dificultad de establecer un concepto que aúne las diferentes visiones existentes en el ámbito investigador y que pueda adaptarse a los continuos y vertiginosos cambios tecnológicos. Pese a ello, desde diversas instituciones se ha impulsado proyectos tendentes a establecer estándares de referencia que pudieran ser utilizados en contextos sociales y entornos geográficos diversos, entre los que destaca el marco europeo DigComp (Carretero et al., 2017). Sus recomendaciones han sido tenidas en cuenta en el diseño de alguno de los instrumentos más recientes y, a día de hoy, parece el mejor marco de referencia a seguir, si bien su pervivencia dependerá de su capacidad de adaptación a una realidad cada vez más cambiante.

El predominio del cuestionario de autoevaluación como tipo de herramienta provoca que los procesos de validación sean necesarios para eliminar el sesgo que se deriva de la autopercepción de una determinada habilidad. Por ello, se ha de destacar el trabajo realizado por Esteve (2015) con la herramienta ETeach3D puesto que ofrece una vía para el contraste de los cuestionarios con pruebas que pueden resultar más objetivas a la hora de valorar la destreza en el uso de las TIC. De hecho, los resultados derivados de esta investigación indican que la población estudiada tiende a valorar su nivel de competencia digital por encima de sus verdaderas capacidades, así que parece conveniente el desarrollo de instrumentos de medida que puedan complementar la información obtenida mediante el autodiagnóstico.

En cuanto a la estructura de los cuestionarios, además de la recopilación inicial de datos identificativos, la mayor parte de ellos comparten ítems que tienen que ver con el uso y disponibilidad, con el conocimiento de herramientas concretas y con la actitud mostrada hacia las TIC. Suele existir además apartados vinculados con la seguridad digital y con el tratamiento de la información, si bien estas cuestiones son tratadas con mayor profundidad en los instrumentos más recientes. Pese a que casi todos optan por preguntas de respuesta numérica y al predominio del soporte digital, la extensión de los cuestionarios es muy variable y sólo uno de ellos, el Test Ikanos, incorpora una retroalimentación automática en forma de informe con el perfil digital de cada usuario que resulta muy útil.

No obstante, a pesar de la cantidad de información recopilada, se ha de señalar que no siempre resulta sencillo el acceso a la misma, especialmente a la relacionada con el contenido de los cuestionarios y sus ítems. Este aspecto choca con los parámetros de transparencia que se definen en la denominada Sociedad de la Información y el Conocimiento y con el trabajo colaborativo que se defiende en muchas instituciones educativas.

Finalmente, como ya se ha expuesto, se debe lamentar la escasez de investigaciones realizadas en entornos educativos no formales e informales y en contextos de dificultad social como el abordado por Carrera et al. (2011). Se trata de un campo poco trabajado y podría resultar interesante el desarrollo de iniciativas que estudien la relación entre el nivel de competencia digital y las situaciones de exclusión social.

\section{ENLACES}

Las tablas con los datos recogidos y otras informaciones de interés relacionadas con este trabajo se pueden encontrar en el siguiente enlace: https://bit.ly/3950zE6 


\section{REFERENCIAS BIBLIOGRÁFICAS}

Ala-Mutka, K. (2011). Mapping Digital Competence: Towards a Conceptual Understanding. Publication Office ot the European Union https://bit.ly/2UPRLJW

Carrera, F. X., Vaquero, E., y Balsells, M. A. (2011). Instrumento de evaluación de competencias digitales para adolescentes en riesgo social. EDUTEC: Revista electronica de tecnologia educativa, 35, 1-25. https://bit.ly/3fjyius

Carretero, S., Vuorikari, R. y Punie, Y. (2017). DigComp 2.1. The digital Competence Framework for Citizens. With eight proficiency levels and examples of use. Publication Office of the European Union. https://bit.ly/3dYdA1q

Colás, M.P., Conde, J. y Reyes, S. (2017). Competencias digitales del alumnado no universitario. RELATEC. Revista Latinoamericana de Tecnología Educativa, 16(1). https://doi.org/10.17398/1695-288x.16.1.7

Cózar, R. y Roblizo, M. (2014). La competencia digital en la formación de los futuros maestros: percepciones de los alumnos de los Grados de Maestro de la Facultad de Educación de Albacete. RELATEC: Revista Latinoamericana de Tecnología Educativa, 13(2), 119-133. https://doi.org/10.17398/1695-288X.13.2.119

De Pablos, J., Colás, P., Conde, J. y Reyes, S. (2016). La competencia digital de los estudiantes de educación no universitaria: variables predictivas. Bordón. Revista de Pedagogía, 69(1), 169-185. https://doi.org/10.13042/bordon.2016.48594

Espuny, C., González, V. y Gisbert, M. (2010). ¿Cuál es la competencia digital del alumnado al llegar a la Universidad? Datos de una evaluación cero. Enseñanza \& Teaching. Revista Interuniversitaria de Didáctica, 28(2), 113-137. https://bit.ly/3fnMQcp

Esteve, F. (2015). La competencia digital docente: análisis de la autopercepción y evaluación del desempeño de los estudiantes universitarios de educación por medio de un entorno 3D [tesis doctoral, Universitat Rovira i Virgili]. TDX. https://bit.ly/3kWVmil

Esteve, F., y Gisbert, M. (2013). Competencia digital en la educación superior: instrumentos de evaluación y nuevos entornos. Enl@ce: Revista Venezolana de Información, Tecnología y Conocimiento, 10(3), 29-43. https://bit.ly/2ULSf3H

Fernández, Á. D. (2018) La competencia digital del alumnado de educación secundaria en el marco de un proyecto educativo TIC (1:1). EDUTEC, Revista electrónica de Tecnología Educativa, 63, 60-72. https://doi.org/10.21556/edutec.2018.63.1027

Gisbert, M., Espuny, C. y González, J. (2011). INCOTIC. Una herramienta para la @utoevaluación diagnóstica de la competencia digital en la universidad. Profesorado. Revista de Currículum y Formación de Profesorado, 15(1), 75-90. https://bit.ly/3kXTIUI

Gisbert, M. y Esteve, F. (2011). Digital Leaners: la competencia digital de los estudiantes universitarios. La Cuestión Universitaria, 7, 48-59. https://bit.ly/2HnW0ZU

González, J. (2012). El proyecto eduCAT1x1 y su impacto en la asignatura de Lengua Castellana [tesis doctoral , Universidad Rovira i Virgili]. TDX. https://bit.ly/3fomYx8

González, J., Espuny, C., de Cid, M. J. y Gisbert, M. (2012). INCOTIC-ESO. Cómo autoevaluar y diagnosticar la competencia digital en la Escuela 2.0. Revista de Investigación Educativa, 30(2), 287-302. https://doi.org/10.6018/rie.30.2.117941 
González, J., Espuny, C. y Gisbert, M. (2010). La evaluación cero de la competencia nuclear digital en los nuevos grados del EEES. @tic. Revista d'innovació educativa, 4, 13-20. https://bit.ly/338y8RM

González, J., Esteve, F. M., Larraz, V., Espuny, C. y Gisbert, M. (2018). INCOTIC 2.0. Una nueva herramienta para la autoevaluación de la competencia digital del alumnado universitario. Profesorado. Revista de Currículum y Formación de Profesorado, 22(4), 133-152. https://doi.org/10.30827/profesorado.v22i4.8401

González, V. y Román, M.(2016).Investigador novel: estrategias y herramientas en un mundo conectado. RIITE. Revista Interuniversitaria de Investigación en Tecnología Educativa, 0, 5108. http://dx.doi.org/10.6018/riite/2016/262241

González, V., Román, M., y Prendes, M.P. (2018). Formación en competencias digitales para estudiantes universitarios basada en el modelo DigComp. EDUTEC. Revista Electrónica de Tecnología Educativa, 65, 1-15. https://doi.org/10.21556/edutec.2018.65.1119

ISTE (2007). NETS for Students. https://bit.ly/33sGljE

Larraz, V. (2013). La competència digital a la Universitat. [Tesis doctoral, Universitat d'Andorra]. TDX. https://bit.ly/3kRarpa

Larraz, V., Yáñez, C., Gisbert, M. y Espuny, C. (2013). Modelo para el desarrollo y acreditación de la competencia digital en la universidad. XVI Congreso Internacional EDUTEC 2013. Costa Rica. https://bit.ly/2J48Ay8

Mirete, A. B., García, F. A. y Hernández, F. (2015). Cuestionario para el estudio de la actitud, el conocimiento y el uso de TIC (ACUTIC) en Educación Superior. Estudio de fiabilidad y validez. Revista interuniversitaria de formación del profesorado, 83, 75-89. https://bit.ly/3lWggRn

Pech, S. J. y Prieto, M. E. (2016). La medición de la Competencia Digital e Informacional. En T. De Elón, J. García y E. Orozco (Ed.), Desarrollo de las competencias para el Siglo XXI (pp. 26-50). Humboldt Group Foundation. https://bit.ly/3pQma98

Pérez, A. (2015). Alfabetización Digital y Competencias Digitales en el Marco de la Evaluación Educativa: Estudio en Docentes y Alumnos de Educación Primaria en Castilla y León [tesis doctoral, Universidad de Salamanca]. Gredos. https://bit.ly/2IZrFBk

Prendes, M.P., Castañeda, L. y Gutiérrez-Porlán, I. (2010). Competencias para el uso de TIC de los futuros maestros. Comunicar, 35, 175-182. https://doi.org/10.3916/C35-2010-03-11

Comisión Europea (2006). Recomendación del Parlamento Europeo y del Consejo de 18 de diciembre de 2006, sobre las competencias clave para el aprendizaje permanente. Diario Oficial de la Unión Europea. https://bit.ly/3pQt8e5

Roblizo, M., y Cózar, R. (2015). Usos y competencias en tic en los futuros maestros de educación infantil y primaria: hacia una alfabetización tecnológica real para docentes. Pixel-Bit. Revista de Medios y Educación, 47, 23-39. http://dx.doi.org/10.12795/pixelbit.2015.i47.02

Rodríguez, C., Lorenzo, O. y Herrera, L. (2005). Teoría y práctica del análisis de datos cualitativos. Proceso general y criterios de calidad. Revista Internacional de Ciencias Sociales y Humanidades, SOCIOTAM, 15(2), 133-154. https://bit.ly/35TdzKJ

Roig, R., Rodríguez-Cano, C., Flores, C., Álvarez, J.D., Blasco, J.E., Grau, S. y Tortosa, M.T. (2012). Evaluación de las Competencias Digitales del alumnado en el Espacio Europeo de Educación Superior. En J.D. Álvarez, M.T. Tortosa y N. Pellín (Eds.), X Jornadas de Redes 
de Investigación en Docencia Universitaria: la participación y el compromiso de la comunidad universitaria (pp. 781-795). Universidad de Alicante. https://bit.ly/372p6qs

Vaquero, E. (2013). Estudio sobre la resiliencia y las competencias digitales de los jóvenes adolescentes en situación de riesgo de exclusión social [tesis doctoral, Universitat de Lleida]. TDX. https://bit.ly/36QVNac

Vuorikari, R., Punie, Y., Carretero Gomez S. y Van den Brande, G. (2016). DigComp 2.0: The Digital Competence Framework for Citizens. Update Phase 1: The Conceptual Reference Model. Publication Office ot the European Union. https://bit.ly/337SQ4b

\section{INFORMACIÓN SOBRE LOS AUTORES}

\section{César González Rodríguez}

Universitat de les Illes Balears

Profesor de educación secundaria de la Consejería de Educación del Principado de Asturias, actualmente con destino en el Instituto de Educación Secundaria de Llanes y dentro del Departamento de Tecnología. Anteriormente, durante casi 10 años, trabajé en como educador social en organizaciones del tercer sector.

Cursando en la actualidad el Máster Universitario en Tecnología Educativa: e-learning y Gestión del Conocimiento de URV, UdL, UM y UIB con desarrollo de líneas de investigación sobre la medida del nivel de competencia digital.

Finalizados estudios de Máster Universitario en Formación del Profesorado de Educación Secundaria Obligatoria, Bachillerato y Formación Profesional (UNED), Grado en Economía (UNED), Diplomatura en Educación Social (UNED) e Ingeniería Química (Universidad de Oviedo).

\section{Santos Urbina Ramírez}

Universitat de les Illes Balears

Profesor titular del Área de Didáctica y Organización Escolar del Departamento de Ciencias de la Educación de la Universidad de las Islas Baleares, impartiendo asignaturas relacionadas con la Tecnología Educativa, en diferentes estudios de la Facultad de Educación de esta universidad. Profesor del máster y doctorado interuniversitario en Tecnología Educativa, organizado por las siguientes universidades: URV, UdL, UM y UIB.

Las áreas preferentes de investigación se centran en el diseño, producción y uso de medios didácticos, los entornos de aprendizaje enriquecidos con tecnología y la inserción curricular de las TIC.

Los textos publicados en esta revista están sujetos a una licencia de Reconocimiento 4.0 España de Creative Commons. Puede copiarlos, distribuirlos, comunicarlos públicamente y hacer obras derivadas siempre que reconozca los créditos de las obras (autoría, nombre de la revista, institución editora) de la manera especificada por los autores o por la revista. La licencia completa se puede consultar en:Licencia Creative Commons Atribución-NoComercial-Compartir por igual 4.0 Internacional. 